\title{
Least square fitting with one explicit parameter less
}

\author{
Bernd A. Berg \\ Department of Physics, Florida State University, Tallahassee, FL 32306-4350, USA \\ E-mail: bberg@fsu.edu; Telephone: (850) 644-2868; Fax: (850)644-8630.
}

\begin{abstract}
It is shown that whenever the multiplicative normalization of a fitting function is not known, least square fitting by $\chi^{2}$ minimization can be performed with one parameter less than usual by converting the normalization parameter into a function of the remaining parameters and the data.
\end{abstract}

\section{Program Summary}

Program title: FITM1.

Licensing provisions: none.

Programing language: Fortran 77 with standard extensions (tested with g95 on a Mac).

Operating systems: Any with a Fortran 77 compatible compiler.

$R A M$ required to execute with typical data: 1 Mbyte.

Running time: Less than 1 second on modern PCs.

CPC Library Classification: 4.9 Minimization and Fitting.

Nature of the Problem: Least square minimization when one of the free parameters is the multiplicative normalization of the fitting function.

Solution method: Conversion of the normalization constant into a function of the other parameters and the data, resulting into one explicit fitting parameter less.

Key words: Fitting, Curve Fitting, , Least Square Fitting,

\section{Introduction}

The general situation of fitting by $\chi^{2}$ minimization is that $m$ data points $y_{i}=y\left(x_{i}\right)$ with error bars $\triangle y_{i}$ and a function $y\left(x ; a_{j}\right)$ with $j=1, \ldots, n$ parameters are given and we want to minimize

Preprint submitted to Computer Physics Communications

$$
\chi^{2}=\sum_{i=1}^{m}\left(\frac{y\left(x_{i} ; a_{j}\right)-y_{i}}{\triangle y_{i}}\right)^{2}
$$

with respect to the $n$ parameters, where we neglect (as usual) fluctuations of the $\triangle y_{i}$ error bars.

In many practical application one of the $n$ pa- 
rameters, say $a_{n}$, is the multiplicative normalization of the function $y\left(x ; a_{j}\right)$ so that it can be written as

$y\left(x ; a_{j}\right)=a_{n} f\left(x ; a_{1}, \ldots, a_{n-1}\right)$.

With the parameters $a_{1}, \ldots, a_{n-1}$ fixed there is a unique analytical solution $c_{0}$ for $c=a_{n}$, which minimizes $\chi^{2}(c)$, so that the function $y\left(x ; a_{j}\right)$ depends effectively only on $n-1$ parameters:

$$
\begin{aligned}
& y\left(x ; a_{1}, \ldots, a_{n-1}\right)= \\
& c_{0}\left(a_{1}, \ldots, a_{n-1}\right) \quad f\left(x ; a_{1}, \ldots, a_{n-1}\right) .
\end{aligned}
$$

This can be exploited to perform least square fitting with one parameter less. Remarkably, the number of steps required for one calculation of $\chi^{2}$ remains linear in the number of data.

In section 2 explicit equations for $c_{0}\left(a_{1}, \ldots, a_{n-1}\right)$ and its derivatives with respect to the parameters are derived. Practical examples based on Levenberg-Marquardt fitting $[1,2]$ are given in section 3 . The conclusion from section 3 is that we have an additional useful approach, which mostly converges faster than the corresponding fit with the full number of parameters. Conclusion and an outlook on an eventual application are given in section 4. All examples of this paper can be reproduced with Fortran code that is provided in the Computer Physics Communications software library and documented in our appendix A.

\section{Calculation of the normalization constant and its derivatives}

We find the normalization constant $c_{0}\left(a_{1}, \ldots, a_{n-1}\right)$ by minimizing $\chi^{2}(c), c=a_{n}$ for fixed parameters $a_{1}, \ldots, a_{n-1}$. We define

$\chi^{2}(c)=\sum_{i=1}^{m}\left(\frac{c f\left(x_{i}\right)-y_{i}}{\triangle y_{i}}\right)^{2}$,

so that the derivative with respect to $c$ is zero at the minimum $\chi^{2}\left(c_{0}\right)$ :

$0=\left.\frac{d \chi^{2}}{d c}\right|_{c=c_{0}}=\sum_{i=1}^{m} \frac{2 f\left(x_{i}\right)\left(c_{0} f\left(x_{i}\right)-y_{i}\right)}{\left(\triangle y_{i}\right)^{2}}$
This implies for $c_{0}$ the solution

$$
\begin{gathered}
c_{0}=\frac{r}{s} \text { with } r=\sum_{i=1}^{m} r_{i} y_{i}, \quad r_{i}=\frac{f\left(x_{i}\right)}{\left(\triangle y_{i}\right)^{2}} \\
\text { and } s=\sum_{j=1}^{m} \frac{f\left(x_{j}\right)^{2}}{\left(\triangle y_{j}\right)^{2}} .
\end{gathered}
$$

As it should, this equation reduces for just one data point $(m=1)$ to $c_{0}=y_{1} / f\left(x_{1}\right)$.

For fixed parameters $a_{1}, \ldots, a_{n-1}$ the error bar $\triangle c_{0}$ of $c_{0}$ follows from the variances of the data points:

$$
\left.\left(\triangle c_{0}\right)^{2}\right|_{a}=s^{-2} \sum_{i=1}^{m}\left(r_{i} \triangle y_{i}\right)^{2}
$$

where $\left.\right|_{a}$ indicates that the parameters $a_{1}, \ldots, a_{n-1}$ are kept fixed. When all error bars of the data agree, i.e., $\triangle y_{i}=\triangle y$ for $i=1, \ldots, m$ holds, equations (6) and (8) simplify to

$$
\begin{aligned}
c_{0} & =\frac{\sum_{i=1}^{m} y_{i} f\left(x_{i}\right)}{\sum_{j=1}^{m} f\left(x_{j}\right)^{2}} \\
\left.\left(\triangle c_{0}\right)^{2}\right|_{a} & =(\triangle y)^{2} \frac{\sum_{i=1}^{m} f\left(x_{i}\right)^{2}}{\left(\sum_{j=1}^{m} f\left(x_{j}\right)^{2}\right)^{2}} .
\end{aligned}
$$

If, in addition, the function $f(x)$ is a constant, $f\left(x_{i}\right)=f_{0}$ for $i=1, \ldots, m$, we find the usual reduction of the variance through sampling: $\left.\left(\triangle c_{0}\right)^{2}\right|_{a}=\left(\triangle y / f_{0}\right)^{2} / m$. Note that the error bar Eq. (8) does not hold when the parameters $a_{1}, \ldots, a_{n-1}$ are allowed to fluctuate, i.e., have themselves statistical errors $\triangle a_{i}$. Then the propagation of these errors into $c_{0}\left(a_{1}, \ldots, a_{n-1}\right)$ has to be taken into account, which is done below. Eq. (8) is mainly of relevance for the $n=1$ case when $c_{0}$ eliminates the sole parameter $a_{1}$.

In our illustrations based on the LevenbergMarquardt approach as well as for many other fitting methods one needs the derivatives of the fitting function with respect to the parameters. With Eq. (3) they become

$\frac{d y}{d a_{j}}=\frac{d c_{0}}{d a_{j}} f+c_{0} \frac{d f}{d a_{j}}, \quad j=1, \ldots, n-1$.

We find the derivatives of $c_{0}$ from Eq. 6): 


$$
\frac{d s}{d a_{j}}=\sum_{i=1}^{m} \frac{2 f\left(x_{i}\right)}{\left(\triangle y_{i}\right)^{2}} \frac{d f\left(x_{i}\right)}{d a_{j}} \text {. }
$$

Using the derivatives (12) the full variance of $c_{0}$ with the associated error bar $\triangle c_{0}=\sqrt{\left(\triangle c_{0}\right)^{2}}$ becomes

$$
\left(\triangle c_{0}\right)^{2}=\left.\left(\triangle c_{0}\right)^{2}\right|_{a}+\sum_{j=1}^{n-1} \sum_{k=1}^{n-1} \frac{d c_{0}}{d a_{j}} C_{j k} \frac{d c_{0}}{d a_{k}},
$$

where $C_{j k}$ is the covariance matrix of the parameters $a_{1}, \ldots, a_{n-1}$, which is in our examples returned by the Levenberg-Marquardt fitting procedure.

\section{Examples}

In this section we summarize results from least square fits with $n=1$ to $n=4$ parameters $a_{i}, i=$ $1, \ldots, n$, where the last one, $a_{n}$, is always taken to be a multiplicative normalization. The corresponding Fortran code is explained in appendix A.

We apply the Levenberg-Marquardt method in each case to all $n$ parameters as well as to $n-1$ parameters by considering $c_{0}$, the least square minimum of $a_{n}$ given by (6), to be part of the function. The Levenberg-Marquardt method uses steepest decent far from the minimum and switches to the Hessian approximation when the minimum is approached. Our Fortran implementation is a variant of the one of Ref. [3]. Besides the fitting function $y\left(x ; a_{i}\right)$ one has to provide the derivatives $d y / d a_{j}$ and start values for the $n$, respectively $n-1$, parameters $a_{i}$. Usually the method will converge to the nearest local minimum of $\chi^{2}$, i.e., the minimum which has the initial parameters in its valley of attraction.

Our choice of data for which we illustrate the method is rather arbitrary and emerged from considerations of convenience. For the $n=1$ to $n=3$ parameter fits we use deconfining temperatures estimates from Markov Chain Monte Carlo (MCMC) simulations of $4 \mathrm{D} \mathrm{SU}(2)$ gauge theory on $N_{s}^{3} N_{\tau}$ lattices as reported in Table 4 of a paper by Lucini et al. [4]. We aim to extract from them corrections to asymptotic scaling by fitting $\left(a T_{c}\right)^{-1}=N_{\tau}\left(\beta_{c}\right)$ to the form [5]

$$
\begin{aligned}
y & =N_{\tau}(\beta)=\frac{a_{3}}{f_{\lambda}(\beta)} \\
& =\frac{a_{3}}{f_{\lambda}^{a s}(\beta, 2)}\left(1+\frac{a_{2}}{\beta}+\frac{a_{1}}{\beta^{2}}\right),
\end{aligned}
$$

where $N_{\tau}$ is the temporal extension of a $N_{s}^{3} N_{\tau}$ lattice and $f_{\lambda}^{a s}(\beta, N)$ is the universal two-loop asymptotic scaling function of $\mathrm{SU}(\mathrm{N})$ gauge theory

$f_{\lambda}^{a s}(\beta, N)=e^{-1 /\left(2 b_{0} g^{2}\right)}\left(b_{0} g^{2}\right)^{-b_{1} /\left(2 b_{0}^{2}\right)}$

with $g^{2}=2 N / \beta$ and $b_{0}=\left(N / 16 \pi^{2}\right)(11 / 3)$ the one-loop $[6,7], b_{1}=\left(N / 16 \pi^{2}\right)^{2}(34 / 3)$ the two-loop [8,9] result.

In Table 4 of [4] the error bars are for the critical coupling constants $\beta_{c}$. For the purpose of the fit (17) the error bars are shuffled to $N\left(\beta_{c}\right)$ by means of the equation

$\triangle\left(a T_{c}\right)^{-1}=\frac{N_{\tau}}{f_{\lambda}\left(\beta_{c}\right)}\left[f_{\lambda}\left(\beta_{c}\right)-f_{\lambda}\left(\beta_{c}+\triangle \beta_{c}\right)\right]$

where a preliminary estimate of the $f_{\lambda}(\beta)$ scaling function (16) is used. The thus obtained data (omitting $N_{\tau}<4$ lattices) are compiled in Table 1 .

Table 1

Data used for our least square fitting examples.

\begin{tabular}{|c|c|r|c|}
\hline \multicolumn{2}{|c|}{ Lucini et al. [4] } & \multicolumn{2}{|c|}{ Bhanot et al. [10] } \\
\hline$\beta_{c}$ & $N_{\tau}\left(\beta_{c}\right)$ & $N_{s}$ & $\operatorname{Im}(u)$ \\
\hline 2.29860 & $4.0000(77)$ & 4 & $0.087739(5)$ \\
2.37136 & $5.0000(86)$ & 5 & $0.060978(5)$ \\
2.42710 & $6.0000(32)$ & 6 & $0.045411(5)$ \\
2.50900 & $8.0000(32)$ & 8 & $0.028596(5)$ \\
- & - & 10 & $0.019996(5)$ \\
\hline
\end{tabular}

For the $n=4$ parameter fits results from Bhanot et al. [10] for the imaginary part $\operatorname{Im}(u)$ of the partition function zero closest to the real axis are used, which are obtained from MCMC simulation of the 3D Ising model on $N_{s}^{3}$ lattices. These data are also collected in our Table 1.

To leading order their finite size behavior is 
$y=\operatorname{Im}(u)=a_{2} x^{a_{1}}$ with $x=N_{s}$,

where $a_{1}$ is related to the critical exponent $\nu$ of the correlation length by $a_{1}=-1 / \nu$. In the context of our method this 2-parameter fit is of no interest, because it can be mapped onto linear regression for which the $\chi^{2}$ minimum leads to analytical solutions for both parameters, $a_{1}$ and $a_{2}$. For the critical exponent $\nu$ this yields $1 / \nu=1.6185(2)$, but has an unacceptable large $\chi^{2}$, which leads to $Q=0$ for the goodness of fit (details are given in [3]).

One is therefore led to including subleading corrections by moving to the 4-parameter fit

$y=\operatorname{Im}(u)=a_{4} x^{a_{1}}\left(1+a_{2} x^{a_{3}}\right)=a_{4} f(x)(21)$

for which our method replaces $a_{4}$ by $c_{0}$ (6) as function of the other parameters. We are now ready to present the results for our fits.

\subsection{1-parameter fits}

The function (16) reduces to the from

$y=N_{\tau}(\beta)=\frac{a_{1}}{f_{\lambda}^{a s}(\beta)}$

and there are no fitting parameters left when the analytical solution $c_{0}$ (6) with the error bar $\Delta c_{0}$ form (8) is used for $a_{1}$.

Our Levenberg-Marquardt procedure works down to a single fit parameter and uses besides the fitting function (22) the only derivative

$\frac{d y}{d a_{1}}=\frac{1}{f_{\lambda}^{a s}(\beta)}$.

Using the start value $a_{1}=0.0628450$ one finds convergence after three iteration with the results

$a_{1}=0.025336(26)$ and $\chi^{2}=4263$.

Without any iteration identical values for $a_{1}$ and $\chi^{2}$ are obtained by using the analytical solution $c_{0}$ and its error bar (8). Note that $\chi^{2}$ has to be the same for identical parameters. So $c_{0}$ still counts when it comes to counting the degrees of freedom.

Obviously the obtained $\chi^{2}$ is unacceptable large and it is well visible from the 1-par curve in Fig. 1 that this fit is not good. Additional parameters are needed to account for corrections to asymptotic scaling.

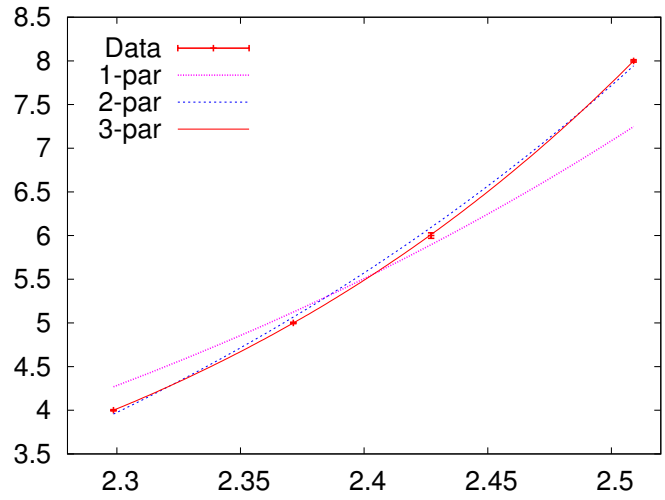

Fig. 1. Uniform distribution: Impossible (left) and missing events (in the enlarged thickness of the right border).

\subsection{2-parameter fits}

The function (16) is now reduced to the form

$y=N_{\tau}(\beta)=\frac{a_{2}}{f_{\lambda}^{a s}(\beta)}\left(1+\frac{a_{1}}{\beta}\right)$.

For the fit with two parameters we use the start values $a_{2}=0.0628450$ (as for $a_{1}$ before) and $a_{1}=$ -1.43424 . After six iterations we find convergence and the results

$\left.a_{1}=-1.5751(84), a_{2}=0.07460(77), \chi^{2}=1126\right)$

Running the fitting routine now just for the parameter $a_{1}$ by eliminating $a_{2}$ in the described way, we find the results (26) after four iterations. In particular, also the error bar of $c_{0}$, now calculated via Eq. (15), agrees with the error bar of $a_{2}$.

Clearly the $\chi^{2}$ is still too large to claim consistency between the fit and the data though the visible improvement is considerable. See the 2-par curve in Fig. 1.

\subsection{3-parameter fits}

We fit now to the full functional form (16). For the three parameter fit the previous starting values are re-shuffled $a_{2} \rightarrow a_{3}, a_{1} \rightarrow a_{2}$ and the additional starting value is taken to be $a_{1}=1$. Our Levenberg-Marquardt procedure needs 245 iterations for convergence and yields the values 
$a_{1}=4.685(83), \quad a_{2}=-4.199(46)$,

$a_{3}=0.395(30), \quad \chi^{2}=0.156$,

which are rather different than the corresponding results $a_{3} \rightarrow a_{2}$ and $a_{2} \rightarrow a_{1}$ (26) of the 2parameter fit.

Identical values (27), (28) are obtained after eliminating the normalization, here $a_{3}$, from the fit parameters used in the Levenberg-Marquardt iteration and we find a reduction of iterations from 245 to 12 .

The $\chi^{2}$ is now small enough to signal consistency between the data and fit, although the 2-par and 3par curves in Fig. 1 are hard to distinguish visually. Converting the $\chi^{2}$ of (28) into a goodness of fit one finds $Q=0.69$.

\subsection{4-parameter fits}

The aim is to perform the 4-parameter fit (21) for the data of Bhanot et al. (Table 1). Using all four parameters, our first set of starting values,

$$
a_{1}=-1.6, \quad a_{2}=0.1, \quad a_{3}=-1.0, a_{4}=0.8,
$$

is chosen so that $a_{1}$ stays close to the 2-parameter fit result $-a_{1}=1 / \nu=1.6185$ (2) from [3]. Running our Levenberg-Marquardt fit program on these initial values, it needs 263,505 iterations to converge and finds

$a_{1}=-1.5981889(17), \quad a_{2}=0.77(22)$,

$a_{3}=-2.81(25), \quad a_{4}=0.79182(85), \quad \chi^{2}=0.1$ (B由)

where the $\chi^{2}$ can be converted into a goodness of fit $Q=0.74$. The huge number of iterations is to some extent accidental, because their number depends for this fit strongly on even small changes of the starting values (we do not pursue this further, because it pertains to the Levenberg-Marquardt method and not to our topic of an alternative fitting approach).

Eliminating the normalization $a_{4}$ from the direct fitting parameters, convergence is reached after 86,922 iterations and we find somewhat different estimates than before:

$a_{1}=-1.5981208(17), a_{2}=0.77(22)$,

$a_{3}=-2.80(25), \quad c_{0}=0.79168(85), \quad \chi^{2}=0.1(B 3)$,
By Murphy's law the important value $a_{1}=-1 / \nu$ seems to have a too small error bars in both fits, while the other (less important) parameters agree well within their error bars and also the second $\chi^{2}$ rounds to the goodness of fit $Q=0.74$. The instability of the result for $1 / \nu$ indicates that the five data points are only marginally sufficient for giving reliable estimates of the four parameters.

In particular, we have a rugged landscape for $\chi^{2}$ as function of the parameters. With different starting values

$$
a_{1}=-4.4, \quad a_{2}=1.3, \quad a_{3}=2.8, \quad a_{4}=0.6
$$

a minimum entirely different from the one above was found by accident. Running our LevenbergMarquardt procedure on these starting values convergence is found after 231 iterations and gives

$$
\begin{aligned}
& a_{1}=-4.40000000265(56), \quad a_{2}=1.303(65),(33) \\
& a_{3}=2.8019(13), \quad a_{4}=0.608(29), \quad \chi^{2}=0.1(34)
\end{aligned}
$$

Eliminating the normalization $a_{4}$ we find convergence after 572 iterations (for the first time an increase) and compatible results:

$a_{1}=-4.40000000293(56), \quad a_{2}=1.303(65),(35)$

$a_{3}=2.8019(13), \quad a_{4}=0.608(29), \quad \chi^{2}=0.1($ (B6) $)$

Either set of parameters fits the data perfectly well in their range, while the different fits function diverge quickly out of this range, i.e., for larger lattices.

\section{Conclusion and Outlook}

This paper shows that we can exclude the multiplicative normalization of a fitting function from the variable parameters of a $\chi^{2}$ minimization and include it into the fitting function (it still counts when it comes to determining the degrees of freedom). Our simple examples show that this works well, reducing in most cases the number of iterations, though this is not consistently the case.

The code discussed in appendix A shows that there is no extra work involved for the user. Once the general, application independent, code is set up, the subroutine which the user has to supply 
has one parameter less than the one needed for the usual Levenberg-Marquardt fitting procedure (compare the reduction from subg-la3su2.f to suby_la3su2.f). Besides, it is useful to have alternatives at hand when one is trying to find convenient initial values.

Finally, there may be interesting applications, which cannot be easily incorporated into conventional fitting schemes. For instance, there are situations where distinct data sets are supposed to be described by the same function with different multiplicative normalizations. An example is scale setting in lattice gauge theories [11]. The method of this paper can then be used to eliminate all multiplicative constants from the independent parameters of the fit so that one can consolidate all data sets into one fit. This application requires some extension of the code of the present paper and has been already been used in Ref. [12].

Acknowledgments: This work was in part supported by the US Department of Energy under contract DE-FG02-13ER41942. After posting this paper Georg von Hippel informed me kindly of a closely related method published some time ago by Golub and Pereya [13]. Our Fortran implementation provides a good starting point for newcomers to these methods.

\section{Appendix A. Fortran implementation}

The Fortran code can be downloaded from the Computer Physics Communications software library as and archive file FITM1.tgz, which unfolds under

$$
\text { tar - zxvf FITM1.tgz }
$$

into a tree structure with the folder FITM1 at its top. On the next level there are two subfolders examples and libs. To reproduce the results of section 3 go to the examples folder, where one finds the subfolders 1par, 2par, 3par and 4par.

With the exception of 1 par, which is a special case discussed at the end of this appendix, each of these subfolders contains three Fortran programs, $n$ gfit.f, $n$ gfitm1.f and $n$ fitm1.f, where $n=$ 2,3 or 4 denotes the number of parameters. All programs are ready to be compiled and run, say with ./a.out $>$ a.txt. The thus produced results should agree for $n g f i t m 1 . f$ with those found in the text file an.txt and for $n$ gfitm1.f as well as $n$ fitm1.f with those found in anm1.txt. The difference between the last two programs is that the run time of $n$ gfitm1.f scales with $m^{2}$, the square of the number of data, while $n \mathrm{fitm} 1$. f scales linear with $m$ for reasons explained later in this appendix.

In addition graphical output is created. Type

$$
\text { gnuplot gfit.plt }
$$

to see the plots (a gnuplot driver gfit.plt is located in each folder).

Each program reads data and starting values from a provided file named fort.10. For the $n=4$ case there are two sets, bhanot1. dat and bhanot2.dat, which differ by the initial values and the desired one has to be copied on fort. 10 before the run.

The programs $n g f i t . f$ and $n g f i t m 1 . f$ rely both on a Levenberg-Marquardt subroutine named fitsub to find the minimum of $\chi^{2}$. This subroutine, is transferred after the end statement of these programs by an include statement into the code:

include '../../libs/fortran/fitsub.f' .

In the same way this is done for all others routines needed so that the include statements allow for easy tracking of the location of the source code of each routine.

The crucial difference between these two main programs is: $n$ gfit. $f$ calls $f i t s u b$ for $n f i t=n$ parameters, but $n$ gfitm 1 calls it for $n f i t=n-1$ parameters. This is achieved by feeding distinct subroutines subg into fitsub, which define the fitting functions and their derivatives with respect to the nfit parameters. For instance, for the 3 -parameter case the subroutine used by the run of $3 g f i t . f$ is subg_la3su2.f as listed here:

subroutine $\operatorname{subg}(x, a, y f i t, d y d a, n f i t)$

c BB May 20 2015. User provided subroutine

c for a 3-parameter fit of the pure su2 scale,

c yfit=a3*(1+a2/x+a1/x**2)/flasu2 $(\mathrm{x}, 2)$ with $\mathrm{x}=$ beta

$c$ and flasu2 $(x, 2)$ the asymptotic su2 scaling

c function (yfit has the dimension of a length). include '../../libs/fortran/implicit.sta'

include '../../libs/fortran/constants.par'

dimension a (nfit), dyda (nfit)

if (nfit.ne.3) stop "subg_la3su2: nfit.ne.3." $\mathrm{x} 2=\mathrm{x} * * 2$

flasu2 $=f l a(x$, itwo $)$

$\operatorname{dyda}(3)=($ one $+a(2) / x+a(1) / x 2) / f l a s u 2$

yfit $=a(3) * \operatorname{dyda}(3)$ 


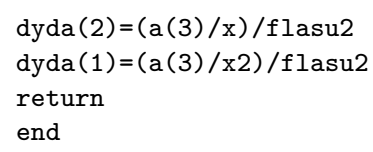

The 3gftim1.f program and all other $n g f i t m 1$.f programs, but $n=1$, include subgfitm 1 . f with the following code:

subroutine subg (xx, aa,yfit,dyda,nfit)

c BB May 20 2015. Generic routine for least square c fit with one parameter less. Input needed: User c provided subroutine suby for unnormalized fit $c$ function and its derivatives with respect to $c$ its parameters.

include '.././libs/fortran/implicit.sta' include '../../libs/fortran/constants.par' include '../../libs/subs/common_fitting.f c For this routine the common transfers $\mathrm{x}, \mathrm{y}$, ye. dimension aa (nfit), dyda (nfit) call chi2dcda (ndat, nfit, $x, y, y e, a a, d c d a, c 0)$ call suby (xx, aa, yy, dyda, nfit) yfit=co*yy

do $i=1$, nfit $\operatorname{dyda}(i)=\operatorname{co} * \operatorname{dyda}(i)+\operatorname{dcda}(i) * y y$ enddo return end

Here the call to suby defines the user supplied function and its derivatives as the subg routines do for the $n$ gfit.f programs. The suby routines replace the normalization parameters by the number one. For our example above subg_la2su2.f becomes suby_la2su2.f given by subroutine suby ( $x, a, y, d y d a, n f i t)$

c BB may 20 2015. User provided fit function

C $y=(1+a 2 / x+a 1 / x * * 2) / f l a s u 2(x, 2)$ and derivatives. include '../../libs/fortran/implicit.sta' include '..././libs/fortran/constants.par' dimension a(nfit), dyda (nfit)

if(nfit.ne.2) stop "suby_la3su2: nfit.ne.2." $\mathrm{x} 2=\mathrm{x} * * 2$

flasu2 $=f l a(x$, itwo $)$

$\mathrm{y}=($ one $+\mathrm{a}(2) / \mathrm{x}+\mathrm{a}(1) / \mathrm{x} 2) / \mathrm{flasu} 2$

$\operatorname{dyda}(2)=($ one $/ x) / f l$ asu 2

$\operatorname{dyda}(1)=($ one $/ \mathrm{x} 2) / \mathrm{flasu} 2$

return

end

This it the only routine, which the user has to define for the reduced fitting procedure. The chi2dcda routine called in subgfitm1.f is generic for all these fits and calculates $c_{0}(6)$ and its derivatives (12) as defined in section 2 with the following code:

subroutine chi2dcda ( $, \mathrm{nf}, \mathrm{x}, \mathrm{y}, \mathrm{ye}, \mathrm{a}, \mathrm{dcda}, \mathrm{c0})$

c BB May 20 2015. Determination of the normalization $c$ constant $\mathrm{c} 0$ for chi2 fit of data $y_{-} i$ with the fit

c function $\mathrm{c} 0 * \mathrm{f}$. Then, in dcda the derivatives of c c0 with respect to the fit parameters.

c Input: data arrays $x(n), y(n)$, ye (n),

c parameter array a(nf).

c Output: dcda(nf), c0.

c variable fit parameters a(nf).

include '.././libs/fortran/implicit.sta'

include '.././libs/fortran/constants.par' parameter $(\mathrm{mf}=30)$ ! maximum number parameters. dimension drda(mf), dsda(mf), dfda(mf), a (nf),

$\& \operatorname{dcda}(n f), x(n), y(n), y e(n)$

if (nf.gt.mf) stop "chi2dcda: nf.gt.mf."

$r=$ zero

$\mathrm{s}=$ zero

do $j=1, n f$

$\operatorname{drda}(j)=$ zero

$\operatorname{dsda}(j)=$ zero

enddo

do $i=1, n$

call suby ( $x(i), a, f, d f d a, n f)$

ye2i=one/ye (i) $* * 2 ! 1 /$ (error bar squared).

$r i=f * y e 2 i$

$r=r+y(i) * r i \quad$ ! iterate $r$.

$\mathrm{s}=\mathrm{s}+\mathrm{f} * \mathrm{r} \quad$ ! iterate $\mathrm{s}$

addy $=y(i) * y e 2 i$

addf $=$ two $* r i$

do $j=1, n f$

$\operatorname{drda}(j)=\operatorname{drda}(j)+\operatorname{addy} * \operatorname{dfda}(j)$

$\operatorname{dsda}(j)=\operatorname{dsda}(j)+\operatorname{addf} * \operatorname{dfda}(j)$

enddo

enddo

$\mathrm{c} 0=\mathrm{r} / \mathrm{s}$

do $j=1, n f$

$\operatorname{dcda}(j)=(s * \operatorname{drda}(j)-r * \operatorname{dsda}(j)) / s * * 2$

enddo

return

end

An extension of this routine, chi2c0eb.f, calculates also the variance (15) of $c_{0}$ and needs on input the covariance matrix of the parameters $a_{1}, \ldots, a_{n-1}$.

Unfortunately, calling chi2dcda in subgfitm1.f leads to a $\mathrm{m}^{2}$ scaling with the number of data, because the fitsub. $f$ routine makes per iteration already $m$ calls to subg, one for each data point. This is resolved by moving the chi2dcda call into the fitting routine, just before the loop in which it calls subg. The nfitm1.f programs use the thus modified Levenberg-Marquardt routine and use

include '../../libs/fortran/fitm1sub.f'

together replacing the subroutine subgfitm $1 . f$ by a modification, subfitm1.f.

Finally, the program $1 \mathrm{fitm} 1 . \mathrm{f}$ in 1 par is a special case, because we have nfit $=0$ parameters left and a fitting routine is not needed for this case. It 
becomes replaced by a variant of the chi2dcda.f routine: chi2c0.f calculates $c_{0}(6)$ and its error bar, now according to Eq. (8) instead of (15).

\section{References}

[1] K. Levenberg, Qty. Appl. Math. 2 (1944) 164.

[2] D.W. Marquardt, SIAM 11 (1963) 431.

[3] B.A. Berg, Markov Chain Monte Carlo Simulations and Their Statistical Analysis, World Scientific, Singapore 2004.

[4] B. Lucini, M. Teper and U. Wenger, JEHP 1 (2004) 61.

[5] The $a_{2}$ and $a_{1}$ parameters are some of the corrections contained in the functional form proposed by A. Bazavov, B.A. Berg and A. Velytsky, Phys. Rev. D 74 (2006) 014501.

[6] D.J. Gross and F. Wilczek, Phys. Rev. Lett. 30 (1973) 1343.

[7] D. Politzer, Phys. Rev. Lett. 30 (1973) 1346.

[8] D.R.T. Jones, Nucl. Phys. B 75 (1974) 531.

[9] W. Caswell, Phys. Rev. Lett. 33 (1974) 244.

[10] G. Bhanot, R. Salvador, S. Black, P. Carter and R. Toral, Phys. Rev. Lett. 59 (1987) 803.

[11] For a review see: R. Sommer, POS (Lattice 2013) 015.

[12] B.A. Berg, Phys. Rev. D 92 (2015) 054501.

[13] G.H. Golub and V. Pereya, SIAM J. Numer. Anal. 2 (1972) 413. 